\title{
High Intensity Interval Training Ameliorates Mitochondrial Dysfunction in the Left Ventricle of Mice with Type 2 Diabetes
}

Fredrik H. Bækkerud ${ }^{1}$, Simona Salerno ${ }^{1}$, Paola Ceriotti ${ }^{2,3}$, Cecilie Morland ${ }^{4}$, Jon Storm-Mathisen ${ }^{5}$, Linda H. Bergersen ${ }^{6,7}$, Morten A. Høydal' ${ }^{8,9}$, 'Daniele Catalucci' ${ }^{2,3}$, Tomas O. St $\left.\varnothing\right|^{\prime} n^{1,8,9}$

Affiliations: p12

\begin{abstract}
Both human and animal studies have shown mitochondrial and contractile dysfunction in hearts of type 2 diabetes mellitus (T2DM). Exercise training has shown positive effects on cardiac function, but its effect on the mitochondria have been insufficiently explored. The aim of this study was to assess the effect of exercise training on mitochondrial function in T2DM hearts. We divided T2DM mice $(\mathrm{db} / \mathrm{db})$ into a sedentary and an interval training group at 8 weeks of age and used heterozygote $\mathrm{db} /+$ as controls. After 8 weeks of training, we evaluated mitochondrial structure and function, as well as the levels of mRNA and proteins involved in key metabolic processes from the left ventricle. $\mathrm{db} / \mathrm{db}$ animals showed decreased oxidative phosphorylation capacity and fragmented mitochondria. Mitochondrial respiration showed a blunted response to $\mathrm{Ca}^{2+}$ along with reduced protein levels of the mitochondrial calcium uniporter. Exercise training ameliorated the reduced oxidative phosphorylation in complex (C) I + II, Cll and CIV, but not $\mathrm{Cl}$ or $\mathrm{Ca}^{2+}$ response. Mitochondrial fragmentation was partially restored. mRNA levels of isocitrate, succinate and oxoglutarate dehydrogenase were increased in $\mathrm{db} / \mathrm{db}$ mice and normalized by exercise training. Exercise training induced an upregulation of two transcripts of peroxisome proliferator activated receptor gamma coactivator 1 alpha (PGC1 1 and PGC1a4) previously linked to endurance training adaptations and strength training adaptations, respectively. The T2DM heart showed mitochondrial dysfunction at multiple levels and exercise training ameliorated some, but not all mitochondrial dysfunctions.
\end{abstract}

Keywords Mitochondria · Exercise training · Diabetes · Diabetic cardiomyopathy

\begin{tabular}{ll}
\multicolumn{2}{l}{ Abbreviations } \\
A & Late LV filling \\
C & Mitochondrial electron transport chain complex (ex. ClI = complex II) \\
E & Early LV filling \\
E $^{\prime}$ & Myocardial velocity \\
EF & Ejection fraction \\
ETC & Electron transport chain \\
HIIT & High intensity interval training \\
IDH & Isocitrate dehydrogenase \\
LV & Left ventricle \\
MCU & Mitochondrial calcium uniporter OGDH Oxoglutarate dehydrogenase \\
OXPHOS & Oxidative phosphorylation \\
PGC1- $\alpha$ & Peroxisome proliferator-activated receptor gamma coactivator 1-alpha \\
PPARY & Peroxisome proliferator-activated receptory \\
SDH & Succinate dehydrogenase \\
T2DM & Type 2 diabetes mellitus \\
TFAM & Mitochondrial transcription factor A
\end{tabular}

Handling Editor: Y. James Kang.

Daniele Catalucci and Tomas O. Stølen contributed equally to this work.

Electronic supplementary material The online version of this article (https://doi.org/10.1007/s12012-019-09514-z) contains supplementary material, which is available to authorized users.

Correspondence: Fredrik H. Bækkerud fredrik.h.bakkerud@ntnu.no

Extended author information available on the last page of the article 


\section{Background}

Improved medical treatment has increased life expectancy and reduced years lost due to illness among most diseases with the exception of diabetes mellitus and some neoplasms [1]. Since cardiovascular disease is the leading cause of death among patients with type 2 diabetes mellitus (T2DM) [2-5], there is great interest in examining the changes that occur in the T2DM heart and their potential therapies.

Mitochondrial dysfunction is a key feature in T2DM hearts, and reduced respiratory capacity and efficiency contribute to the reduced contractility in diabetic hearts [6]. Substrate switch and oxygen wasting [6], reduced oxidative phosphorylation (OXPHOS) of Complex I, III and IV [7, 8], and altered mitochondrial morphology [8] are reported in T2DM hearts.

In addition to lower OXPHOS capacity, the T2DM heart likely experiences a dysfunctional regulation of OXPHOS due to $\mathrm{Ca}^{2+}$ dysregulation. $\mathrm{Ca}^{2+}$ is an important regulator of mitochondrial respiration, activating enzymes of the Krebs cycle as well as the mitochondrial electron transport chain (ETC) $[9,10]$. Mitochondrial $\mathrm{Ca}^{2+}$ sensing is one of the mechanisms of how excitation-contraction coupling interacts with the mitochondria to adapt to an increased energy demand. The mitochondrial calcium uniporter $(\mathrm{MCU})$ is the main source of mitochondria $\mathrm{Ca}^{2+}$ uptake and has recently been implicated in the mitochondrial and cardiac responses to stress [11, 12]. MCU knockout leads to lower mitochondrial $\mathrm{Ca}^{2+}$ uptake and a slower response of metabolism and contractility during stress with beta adrenergic agonists [11]. Additionally, MCU knockout impairs the heart's metabolic and contractile response to increasing heart rate by pacing [12]. Streptozotocin-induced diabetic mice show reduced expression of $\mathrm{MCU}[13,14]$, and in vitro studies indicate that it is the hyperglycemia which cause the reduction in $\mathrm{MCU}$ and reduced intramitochondrial $\mathrm{Ca}^{2+}$ concentration [13].

Low cardiorespiratory fitness (maximal oxygen uptake; $\mathrm{VO}_{2 \max }$ ) is one of the strongest predictors for cardiovascular death in a range of diseases including T2DM [15], and exer-cise training is therefore regarded as beneficial. Clinical and epidemiological studies indicate that only minor changes in $\mathrm{VO}_{2 \max }$ improve cardiovascular health and survival [16-18]. However, the largest effects have been observed after high intensity aerobic training [17, 19, 20]. High intensity interval training (HIIT) in T2DM patients improves $\mathrm{VO}_{2 \max }$ and diastolic function more than moderate intensity exercise training [21]. Exercise training restores contractility in T2DM mice and streptozotocin induced diabetic rats. This is partly due to restored

$\mathrm{Ca}^{2+}$ handling $[22,23]$ and increased mitochondrial biogenesis through peroxisome proliferator activated receptor gamma coactivator 1 alpha (PGC-1 $\alpha$ ) and protein kinase B activation [24]. Still, the effects of exercise training on the different ETC complexes and MCU in the T2DM heart are largely unknown.

The aim of this study was to examine the effect of HIIT on mitochondrial OXPHOS capacity in the $\mathrm{db} / \mathrm{db}$ mouse model of T2DM. In addition, we investigated the effects of T2DM and HIIT on the mitochondrial response to $\mathrm{Ca}^{2+}$. We hypothesized that HIIT would rescue an impaired mitochondrial OXPHOS capacity in the left ventricle (LV) of $\mathrm{db} / \mathrm{db}$ mice and reverse T2DM induced changes in mitochondrial size and quantity. Further we hypothesized that the mitochondrial response to $\mathrm{Ca}^{2+}$ would be restored with exercise training in $\mathrm{db} / \mathrm{db}$ mouse hearts.

\section{Methods}

\section{Animals}

Thirty male T2DM db/db (BKS.Cg-m +/+ Lepdb/Bom Tac) and 17 heterozygote db/+ lean healthy controls (BKS.Cg-m +/+ Lepdb/+ lean) were used in these experiments (Taconic Biosciences). The homozygote $\mathrm{db} / \mathrm{db}$ animals develop diabetes due to overeating resulting from a mutation in the leptin receptor [25]. The $\mathrm{db} / \mathrm{db}$ animals were divided into one high intensity interval training group and one sedentary group ( $n=15$ each group), while heterozygote $\mathrm{db} /+$ served as healthy controls. The animals were allocated to cages by staff at the Comparative Medicine Core Facility at the Norwegian University of Science and Technology. Baseline testing of $\mathrm{VO}_{2 \max }$ and echocardiography were done at 8 weeks of age before the animals started their 8 week train-ing regime. After the exercise training period, at 16 weeks of age, baseline tests were repeated before the animals were euthanized and tissue was harvested. The mice were kept on a $12 \mathrm{~h}$ light/dark cycle and had access to food and water ad libitum. The study was approved by the Norwegian council for animal research. One $\mathrm{db} / \mathrm{db}$ and one $\mathrm{db} /+$ animal died during the follow-up. The $\mathrm{db} / \mathrm{db}$ animal died before exercise training was started. 


\section{$\mathrm{VO}_{2 \max }$ and Exercise Training}

The $\mathrm{VO}_{2 \max }$ testing and exercise training protocols used in this study have been described extensively elsewhere [26]. In short, animals ran on a customized treadmill inside a metabolic chamber at $25^{\circ}$ inclination with gradually increasing speed every minute by $0.03 \mathrm{~m} / \mathrm{s}$ until exhaustion. Animals were exercised from 8 to 16 weeks of age. The training consisted of treadmill running, starting with a 10-min warm up before ten intervals of 4 min each were performed at $90 \%$ of $\mathrm{VO}_{2 \max }$ interspaced by 2 min at $70 \%$ of $\mathrm{VO}_{2 \max }$. This was repeated 5 days a week for 8 weeks.

\section{Mitochondrial Respiration}

The mice were anaesthetized with $4 \%$ isoflurane mixed into a gas mixture originally composed of $70 \% \mathrm{~N}_{2}$ and $30 \% \mathrm{O}_{2}$ through a vaporizer into a chamber before the heart was surgically removed to euthanize the animal. Immediately after euthanization, left ventricular tissue was transferred to a cryotube containing $2 \mathrm{ml}$ ice-cold BIOPS buffer solution [2.77 $\mathrm{mM} \mathrm{CaK}{ }_{2}$ EGTA buffer, $7.23 \mathrm{mM} \mathrm{K}{ }_{2}$ EGTA buffer, $20 \mathrm{mM}$ imidazole, $20 \mathrm{mM}$ taurine, $50 \mathrm{mM}$ 2-( $N$-morpholino) ethanesulfonic acid hydrate, $0.5 \mathrm{mM}$ dithiothreitol, $6.56 \mathrm{mM} \mathrm{MgCl}_{2} \cdot 6 \mathrm{H}_{2} \mathrm{O}, 5.77 \mathrm{mM} \mathrm{Na}_{2}$ ATP and $15 \mathrm{mM} \mathrm{Na}_{2}$ phosphocreatine ( $\mathrm{pH}$ 7.1)]. Tissue sections from the LV were dissected in ice cold BIOPS buffer solution; connective tissue was removed; and fiber bundles were tweezed apart with pre-cooled fine tip forceps. After dissection, the tissue was transferred to $2 \mathrm{ml} \mathrm{BIOPS}$ buffer containing $50 \mu \mathrm{g} / \mathrm{ml}$ saponin for permeabilization while gently agitating at $4{ }^{\circ} \mathrm{C}$ for $20 \mathrm{~min}$, and thereafter washed for 10 min using MIR05 buffer $\left(0.5 \mathrm{mM}\right.$ EGTA, $3 \mathrm{mM} \mathrm{MgCl} \cdot 6 \mathrm{H}_{2} \mathrm{O}, 60 \mathrm{mM}$ lactobionic acid, $20 \mathrm{mM}$ taurine, $10 \mathrm{mM} \mathrm{KH}_{2} \mathrm{PO}_{4}, 20 \mathrm{mM}$ HEPES, $110 \mathrm{mM}$ d-Sucrose, $1 \mathrm{~g} / \mathrm{l} \mathrm{BSA}$ essentially fatty acid free, $\mathrm{pH} \mathrm{7.1)} \mathrm{to} \mathrm{remove} \mathrm{any} \mathrm{excess} \mathrm{saponin,} \mathrm{before} \mathrm{it} \mathrm{was}$ transferred in duplicates to the chambers of the respirometer (Oxygraph-2k, Oroboros, Innsbruck, Austria) containing $2 \mathrm{ml}$ MIR05 at $37^{\circ} \mathrm{C}$.

A titration protocol to measure respiration from Complex I, II and IV (CI, CII and CIV, respectively) was used. Malate $(0.5$ $\mathrm{mM})$, glutamate $(10 \mathrm{mM})$ and pyruvate $(5 \mathrm{mM})$ were already in the chamber to fuel $\mathrm{Cl}$ leak respiration. After addition of the tissue, the oxygen concentration was raised to $\approx 500 \mathrm{nmol} / \mathrm{ml}$ to avoid an oxygen limitation of respiration. Next, ADP (5 $\mathrm{mM}$ ) was added to stimulate $\mathrm{Cl}$ OXPHOS capacity, followed by titrations of succinate $(10 \mathrm{mM})$ for $\mathrm{Cl}+\| \mathrm{OXPHOS}$ and rotenone $(0.5 \mathrm{mM})$ to inhibit $\mathrm{Cl}$ and evaluate $\mathrm{CII}$ OXPHOS. Malonic acid and Antimycin A was added to inhibit $\mathrm{Cll}$ and $\mathrm{CIII}$, the resulting respiration is considered to be extramitochondrial, dubbed residual oxygen consumption. After this step, CIV OXPHOS was evaluated by titrating ascorbate and tetramethyl- $p$-phenylenediamine (TMPD) into the chambers. In addition to stimulating CIV, ascorbate and TMPD together cause oxygen consumption unrelated to the mitochondria. To reveal and correct for this oxygen consumption, we inhibited CIV respiration with Azide and subtracted the resulting oxygen flux from that of Ascorbate and TMPD. All respiration rates were also corrected for residual oxygen consumption. At the end of the protocol, the tissue was extracted from the chambers, blotted on five layers of lens tissue paper to remove excess liquid and weighed on a balance controlled scale (Sartorius ME235P-SD). All respiration values are expressed as pmol $\mathrm{O}_{2} / \mathrm{mg} / \mathrm{s}$. An example trace is shown in Supplementary Fig. 3.

To assess the response of OXPHOS to $\mathrm{Ca}^{2+}$ we prepared the tissue as described above but used a respiration buffer with less $\mathrm{Ca}^{2+}$ chelating agents (in mM: 250 sucrose, 10 Tris $\cdot \mathrm{HCl}, 20$ Tris base, $10 \mathrm{KH}_{2} \mathrm{PO}_{4}, 2 \mathrm{MgCl}_{2} \cdot 6 \mathrm{H}_{2} \mathrm{O}$, and $0.5 \mathrm{mg} / \mathrm{ml}$ $\mathrm{BSA}$ ). Measurements were taken during $\mathrm{Cl}$-linked $\mathrm{OXPHOS}$, as per the protocol above. In pilot experiments, we found $50 \mu \mathrm{M}$ $\mathrm{Ca}^{2+}$ to elicit an increase in OXPHOS and this concentration was used in the experiments. Prior to adding the $50 \mu \mathrm{M} \mathrm{Ca}^{2+}, 50$ $\mu M$ EGTA was added to the chamber to chelate any residual $\mathrm{Ca}^{2+}$ accompanying the substrates to avoid premature calcium effects. Also, $2 \mu \mathrm{M}$ Thapsigargin was added to block $\mathrm{Ca}^{2+}$ uptake by sarcoplasmatic reticulum $\mathrm{Ca}^{2+}$ ATPase. Measurements of mitochondrial respiration were taken blinded to the groups.

\section{Blood Collection}

After the heart was removed, blood was drawn from spillover blood in the thoracic cavity and emptied into a serum tube which was centrifuged at $2000 \times g$ for $10 \mathrm{~min}$. After this, the serum was aliquoted in $500 \mu \mathrm{L}$ aliquots and frozen in liquid nitrogen. The samples were analyzed for glucose and triglycerides by the Medical Biochemistry Department at St. Olavs University Hospital, Trondheim, Norway.

\section{Electron Micrographic Analysis}


The mice were anaesthetized as described for the mitochondrial respiration measurements, before intraperitoneal injection of pentobarbital $(0.2 \mathrm{ml} / 100 \mathrm{~g}$ body weight). Thereafter, the chest was opened, and a 15-gauge needle was inserted into the LV through the apex. A small incision was performed in the right atrium to ensure whole body perfusion. $0.1 \mathrm{M}$ sodium phosphate buffer $(\mathrm{pH} 7.4$ ) containing $4 \%$ paraformaldehyde and $0.1 \%$ glutaraldehyde was perfused for 10 min at a rate of $12-15 \mathrm{ml} / \mathrm{min}$. The cardiac chambers were then dissected, and a small piece of the LV midwall placed in $0.1 \mathrm{M}$ sodium phosphate buffer containing $1 \%$ paraformaldehyde and $2.5 \%$ glutaraldehyde for $24 \mathrm{~h}$ at $4{ }^{\circ} \mathrm{C}$ to optimize electron microscopic morphology and then stored in the same solution diluted 1:10 in $0.1 \mathrm{M}$ sodium phosphate buffer.

Specimens measuring approximately $0.5 \times 0.5 \times 1 \mathrm{~mm}$ were dissected from the LV mid-wall piece and immersed in graded concentrations of glycerol $(10,20$ and $30 \%)$ in $0.1 \mathrm{M}$ phosphate buffer at $4{ }^{\circ} \mathrm{C}$. The samples were exposed to $\mathrm{CaCl}_{2}, 50 \mathrm{mM}$, in $0.1 \mathrm{M}$ sodium cacodylate buffer $\mathrm{pH} 7.4$ at $4{ }^{\circ} \mathrm{C}$ before dehydration in ethanol and embedding in Epon resin. Ultrathin sections (about $70 \mathrm{~nm}$ thickness) were mounted on 300 mesh nickel grids and contrasted by subsequent exposure to uranyl acetate for $15 \mathrm{~min}$ and lead citrate for $90 \mathrm{~s}$, before they were observed in a FEI Tecnai 12 electron microscope. Electron micrographs were taken at a primary magnification of $\times 2550$.

We used ImageJ (National Institute of Health, USA, version 1.50a) to draw the circumference of each mitochondrion on a cross-sectional image taken at $\times 2550$ magnification, with an average of 514 mitochondria per image. Mitochondrial quantity was defined as the number of mitochondrial profiles per $\mu \mathrm{m}^{2}$ of cardiomyocyte area (area of the image after subtracting non-cardiomyocyte area such as blood vessels). Mitochondrial size was defined as the average cross-sectional area $\left(\mu \mathrm{m}^{2}\right)$ of all the mitochondrial profiles (i.e., the total area of mitochondria divided by the number of profiles) encountered in one image. Mitochondrial density was defined as the cross-sectional area of all the mitochondria in one image divided by the cardiomyocyte area. An example trace is shown in Supplementary Fig. 4. Analysis of the EM micrographs was done blinded to the groups.

\section{Western Blot}

Protein expression was evaluated in total lysates by Western blot analyses according to standard procedures. Briefly, samples from mouse LV were homogenized in RIPA buffer [150 mM NaCl, $10 \mathrm{mM}$ Tris pH 7.2, 0.1\% SDS, 1\% Triton-X100, 5 mM EDTA, $100 \mu \mathrm{M} \mathrm{Na}_{3} \mathrm{VO}_{4}, 10 \mathrm{mM} \mathrm{NaF}$, and Protease inhibitor 1X (Thermo Fisher Scientific)] and loaded onto a 4-12\% NuPAGE Tris-Glycine Gel, (Life Technologies), separated by electrophoresis, and transferred to a PVDF membrane (Millipore). Antibodies against the following proteins were used: Mouse anti-MCU (1:1000 dilution) and Rabbit antiTOMM20 (1:10,000 dilution) from Abcam; Goat anti-mouse-HRP (1:5000 dilution) and Goat anti-rabbit-HRP (1:5000 dilution) from Thermo Fisher Scientific. ECL (Millipore) was used for protein detection using a Chemidoc MP Imaging System (Biorad). ImageJ software (National Institutes of Health) was used for densitometry analysis.

\section{RNA Isolation, qPCR and Gene Expression Analysis}

Total RNA was isolated using the RNeasy Mini Kit (Qiagen) and treated with DNase I according to the manufacturer's protocol. cDNA was generated using the Super Script VILO cDNA Syntesis Kit (Life Technologies) and amplified by quantitative real-time PCR using SYBR Green PCR master mix (Applied Biosystems). Relative expression analysis was performed using the $\Delta \Delta C$ t method by normalization to Gapdh. The primers used are listed in Supplementary Table 1.

\section{Cardiac Function}

Cardiac functions were evaluated in vivo via echocardiography (Vevo 2100, VisualSonic) under light anesthesia (2\% isoflurane) using a $40-\mathrm{MHz}$ transducer, on a day of rest from exercise training. The structure of the LV was imaged through $\mathrm{M}$ mode acquisition in the parasternal short axis view and used to derive measurements of heart rate, ejection fraction (EF), stroke volume and cardiac output. Early (E) and atrial (A) filling of the LV were measured by Pulsed Wave Doppler

was placed in line with the mitral valve flow. Finally, myocardial velocity $\left(E^{\prime}\right)$ was measured by Tissue Doppler imaging in the apical four-chamber view by positioning the sample volume at the basal septal segment in the septal wall of the LV. Stress echocardiography was performed after an intraperitoneal injection of dobutamine ( $3 \mathrm{mg} / \mathrm{kg})$. The protocol was repeated once the heart rate was stable.

\section{Statistics}


Group interactions were determined by one way ANOVA. If a significant $F$-value was found, a Tuckey post hoc was used to determine differences among the groups. Changes from 8 to 16 weeks of age $\left(\mathrm{VO}_{2 \max }\right.$ and body weight) were evaluated by a paired $t$ test. SPSS 21 was used for statistical analysis.

\section{Results}

Higher body weight, serum glucose and triglycerides in $\mathrm{db} / \mathrm{db}$ versus $\mathrm{db} /+$ confirm the $\mathrm{db} / \mathrm{db}$ model of T2DM, in addition to a lower $\mathrm{VO}_{2 \max }$. Exercise training lowered body weight and increased $\mathrm{VO}_{2 \max }$, but did not significant affect serum glucose or triglycerides (see Supplementary Figs. 1 and 2).

\section{Mitochondrial Function}

To assess whether exercise training can improve mitochondrial OXPHOS capacity in specific ETC complexes, we employed high-resolution respirometry. OXPHOS capacity in $\mathrm{Cl}, \mathrm{Cl}+\mathrm{II}, \mathrm{Cll}$ and $\mathrm{CIV}$ was decreased by $37,36,37$ and $50 \%$, respectively, in the sedentary $\mathrm{db} / \mathrm{db}$ compared to the $\mathrm{db} /+$ control mice. Exercise trained $\mathrm{db} / \mathrm{db}$ mice had higher OXPHOS capacity for $\mathrm{Cl}+\mathrm{Il}, \mathrm{Cll}$ and $\mathrm{CIV}$ than sedentary db/db (Fig. 1a). We saw no difference in $\mathrm{Cl}$ OXPHOS capacity with exercise training. The $\mathrm{Cll}$ to $\mathrm{Cl}+\| \mathrm{l}$ ratio was $7 \%$ and $9 \%$ higher in exercised $\mathrm{db} / \mathrm{db}$ compared to $\mathrm{db} /+$ control and sedentary $\mathrm{db} / \mathrm{db}$, respectively (Fig. $1 b)$, indicating increased reliance on $\mathrm{Cll}$ even after training. Due to the lower $\mathrm{Cl}$ OXPHOS capacity, the respiratory control ratio for $\mathrm{Cl}$ was also lower by $38 \%$ in $\mathrm{db} / \mathrm{db}$ sedentary and was not higher in exercise trained animals (Fig. 1c). Taken together, these results show that exercise training restored the OXPHOS capacity of $\mathrm{Cl}+\mathrm{Il}, \mathrm{Cll}$ and $\mathrm{ClV}$ in $\mathrm{db} / \mathrm{db}$ hearts.

To explore potential mechanisms for the changes seen in the mitochondria, we examined mRNA of several known transcription factors and coactivators involved in metabolism. PGC1 1 and PGC1 $\alpha 4$ were significantly upregulated in the exercise trained $\mathrm{db} / \mathrm{db}$ mice compared to their sedentary counterparts as well as $\mathrm{db} /+$ controls. The transcription factors peroxisome proliferator-activated receptor $\gamma$ (PPAR $\gamma$ ) and mitochondrial transcription factor A (TFAM) were not

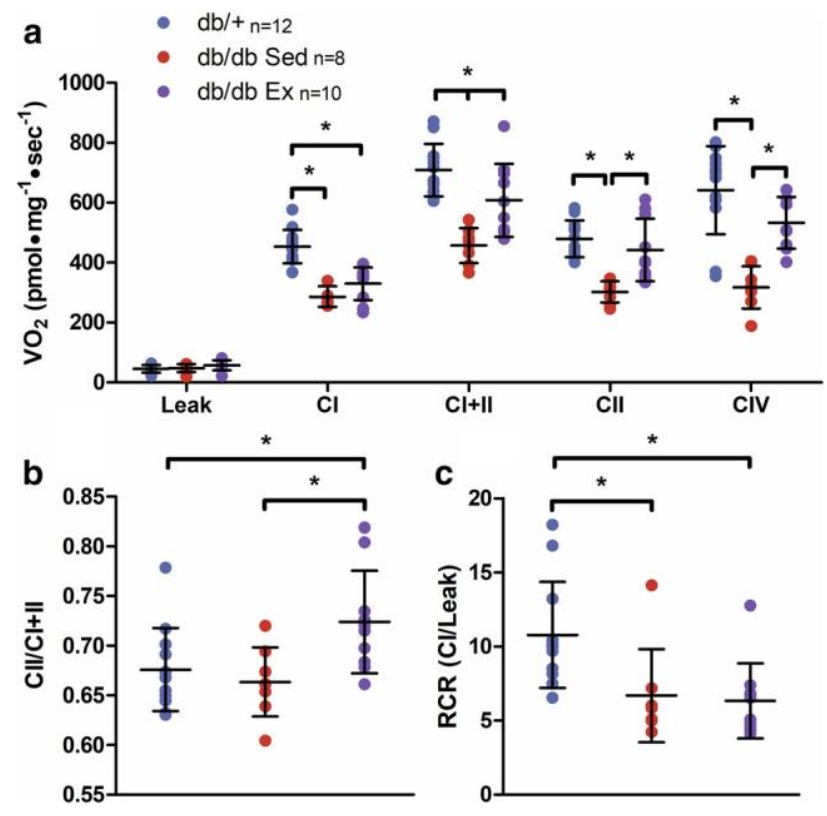

Fig. 1 Exercise training amends the decreased mitochondrial respiratory capacity in $\mathrm{db} / \mathrm{db}$ mice. a Mitochondrial respiration in saponin skinned left ventricular fibers at 16 weeks of age. $\mathbf{b} \mathrm{CII} / \mathrm{Cl}+\|$ ratio, an indication of complex II's contribution to maximal respiration. $\mathbf{c}$ RCR for $\mathrm{Cl}$ $\left(\mathrm{Cl} / \mathrm{Cl}\right.$ leak), an indication of mitochondrial coupling efficiency. $D b /+$ controls, $d b / d b$ Sed diabetic sedentary, $d b / d b$ Ex diabetic exercise trained; ${ }^{*} p$ $<0.05$

for enzymes in the Krebs cycle (isocitrate dehydrogenase, IDH; oxoglutarate dehydrogenase, OGDH; and succinate 
dehydrogenase, $\mathrm{SDH}$ ) were upregulated in $\mathrm{db} / \mathrm{db}$ sedentary compared to $\mathrm{db} /+$ controls. This response was normalized in the exercise trained mice (Fig. 2b).

As exercise training did not uniformly increase OXPHOS capacity, which would be expected with an increased mitochondrial density, we suspected other underlying morphological mechanisms. To investigate this, we determined mitochondrial size, quantity and density using EM images (Fig. 3a-c). The average mitochondrial size was $39 \%$ lower, while mitochondrial quantity was $70 \%$ higher, in $\mathrm{db} / \mathrm{db}$ sedentary than $\mathrm{db} /+$ control mice, leaving mitochondrial density unchanged. In exercise trained $\mathrm{db} / \mathrm{db}$ mice, mitochondrial size and quantity were no longer significantly different from $\mathrm{db} /+$, but not significantly different from $\mathrm{db} / \mathrm{db}$ sedentary $(p=0.098$ and 0.165 , respectively), indicating a partial recovery. There were no changes in mitochondrial density with exercise. To explore a potential activation of genes involved in the fusionfission cycle, we determined mRNA levels of several genes involved in mitochondrial dynamics (Fig. 3e). There were no differences among the groups apart from mitochondrial fission protein 1 , which was upregulated in exercise trained $\mathrm{db} / \mathrm{db}$ mice.

As $\mathrm{Ca}^{2+}$ is an important regulator of mitochondrial func-tion, we wanted to explore how T2DM and exercise training affected the mitochondrial response to $\mathrm{Ca}^{2+}$ (Fig. 4a). Tissue from $\mathrm{db} /+$ mice increased respiration at $50 \mu \mathrm{M} \mathrm{Ca}{ }^{2+}$, but this response was diminished in $\mathrm{db} / \mathrm{db}$ sedentary mice. Exercise training did not influence the mitochondrial response to $\mathrm{Ca}^{2+}$. Along with the depressed response of respiration to $\mathrm{Ca}^{2+}, \mathrm{MCU}$ protein level was decreased in $\mathrm{db} / \mathrm{db}$ mice, but mRNA was unaffected (Fig. $4 b, c)$. In the exercise trained db/db mice, MCU protein levels were higher than sedentary db/db mice.
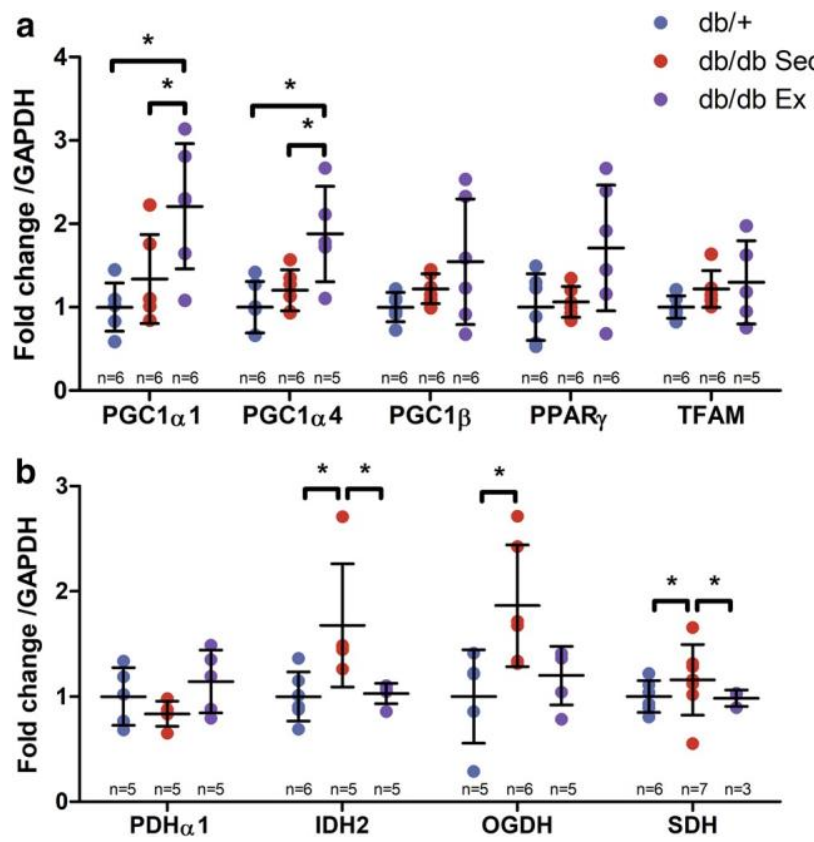

Fig. 2 mRNA of genes involved in metabolism. a mRNA levels of coactivators and transcription factors and of metabolism. $\mathbf{b}$ mRNA levels of genes encoding enzymes of the Krebs cycle. Data from 16 weeks of age. $D b /+$ controls, $d b / d b$ Sed diabetic sedentary, $d b / d b E x$ diabetic exercise trained, GAPDH glyceraldehyde-3-phosphate dehydrogenase, $P G C 1$ peroxisome proliferator activated receptor gamma coactivator

1, PPARY peroxisome proliferator activated receptor gamma, TFAM mitochondrial transcription factor A, PDH 1 pyruvate dehydrogenase alpha 1 , $I D H 2$ isocitrate dehydrogenase-2, $O G D H$ oxoglutarate dehydrogenase, $S D H$ succinate dehydrogenase; $* p<0.05$

\section{Cardiac Function}

Cardiac function was mostly unaltered among groups on most parameters (Table 1). Heart rate was lower in $\mathrm{db} / \mathrm{db}$ sedentary versus $\mathrm{db} /+$ control at 16 weeks of age. Additionally, after injection of the $\beta 1$ receptor agonist dobutamine, a lower heart rate was also observed in exercise trained $\mathrm{db} / \mathrm{db}$ than healthy controls. Stroke volume was not different between groups, but cardiac output was lower in sedentary $\mathrm{db} / \mathrm{db}$ than $\mathrm{db} /+$ controls after dobutamine injection at 16 weeks. There were no changes in the $\mathrm{E} / \mathrm{A}$ or $\mathrm{E} / \mathrm{E}^{\prime}$ ratios at 16 weeks, but mean velocity of blood flow through the mitral valve was lower in $\mathrm{db} / \mathrm{db}$ than $\mathrm{db} /+$. 
a
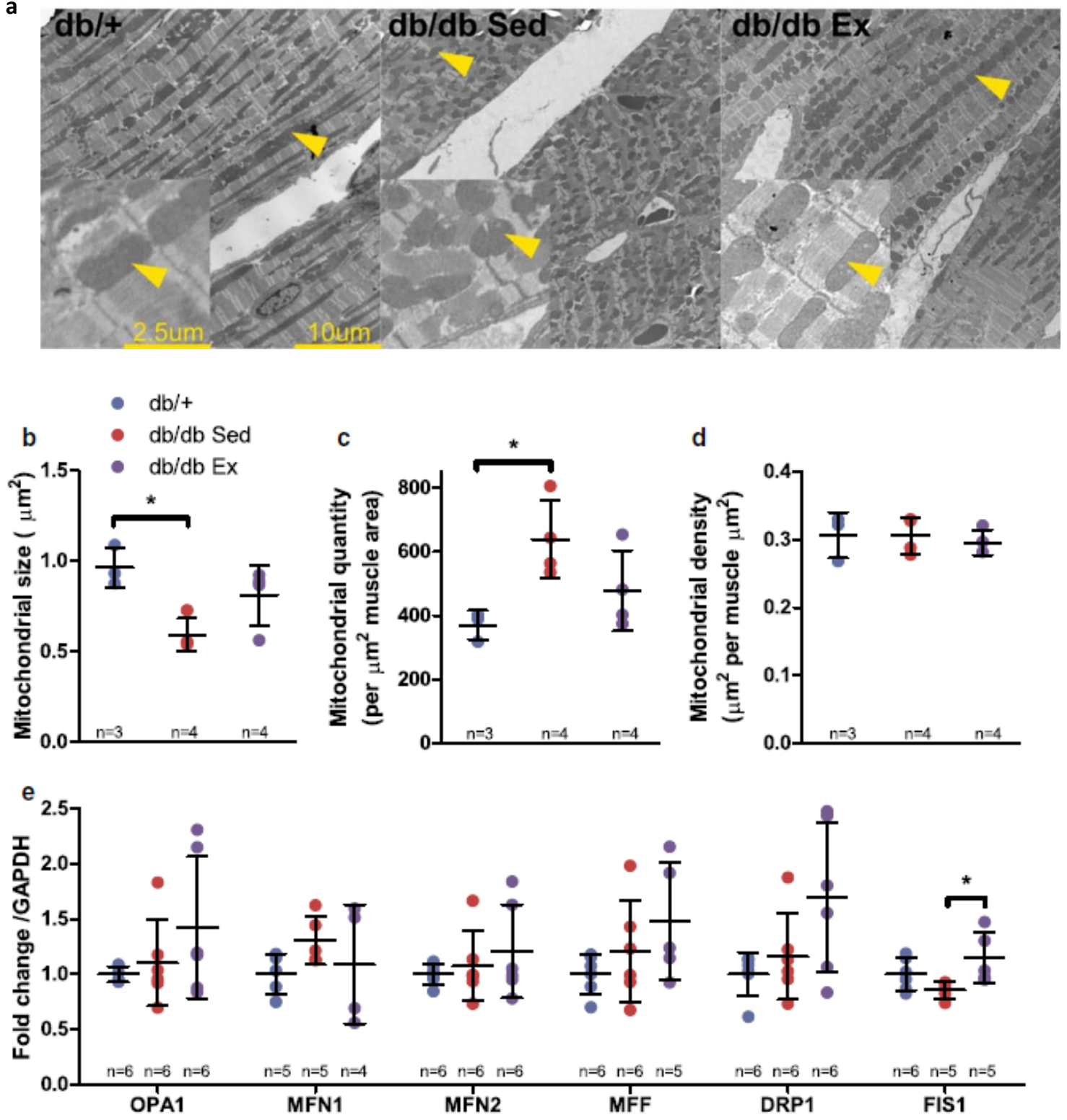

Fig. 3 Mitochondrial fragmentation in $\mathrm{db} / \mathrm{db}$ mice. Mitochondrial morphology from transmission electron micrographs at 16 weeks of age. a Representative electron micrographs, b mitochondrial size (defined as the average area of mitochondrial profiles), c mitochondrial quantity (defined as number of mitochondrial profiles) per $\mu \mathrm{m}^{2}$ of muscle area, $\mathbf{d}$ mitochondrial density (defined as area of mitochondria per cardiomyocyte area), e mRNA of genes involved in mito-chondrial dynamics. Data from 16 weeks of age. $D b /+$ controls, $d b / d b$ Sed diabetic sedentary, $d b / d b$ Ex diabetic exercise trained; yellow arrowheads, examples of mitochondria, OPA1 mitochondrial dynamin like GTPase, MFN mitofusin, MFF mitochondrial fusion factor, DRP1 dynamin like protein 1, FIS1 mitochondrial fission protein 1; ${ }^{*} p<0.05$

\section{Discussion}

Here we show that exercise training ameliorates the impaired left ventricular mitochondrial dysfunction in T2DM. The OXPHOS capacity of CII and CIV in the mitochondrial ETC was restored and $\mathrm{VO}_{2 \max }$ increased in 

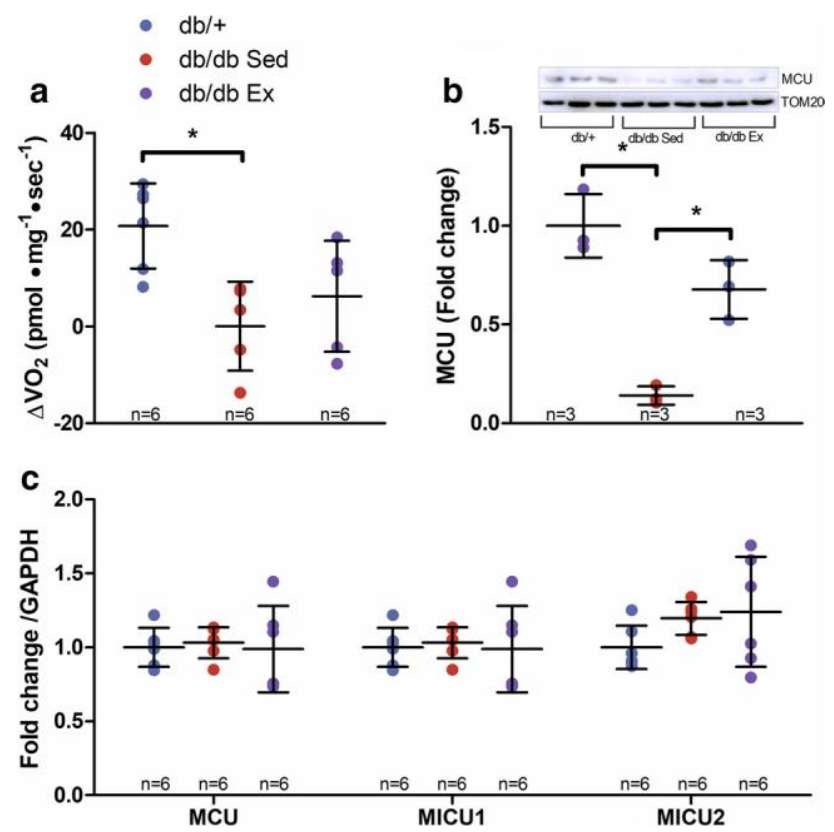

Fig. 4 The mitochondrial respiratory response to calcium is decreased in $\mathrm{db} / \mathrm{db}$ mice. a Change in respiration with addition of $50 \mu \mathrm{M}$ calcium at 16 weeks of age, b MCU protein levels and c MCU mRNA levels. Data from 16 weeks of age. MCU mitochondrial calcium uniporter, GAPDH glyceraldehyde-3-phosphate dehydrogenase; $* p<0.05$

exercise trained $\mathrm{db} / \mathrm{db}$ mice. Mitochondrial morphology appeared fragmented in $\mathrm{db} / \mathrm{db}$, but was not different from control in exercise trained $\mathrm{db} / \mathrm{db}$ mice. The mitochondrial respiratory response to $\mathrm{Ca}^{2+}$ in the $\mathrm{db} / \mathrm{db}$ heart was reduced, along with reduced MCU protein levels. Exercise training was also found to activate not only PGC1 1 , but also PGC1 14 . The latter is a relatively newly discovered transcript of PGC1 $\alpha$ found to be involved in skeletal muscle hypertrophy rather than endurance training adaptations [27]. To our knowledge, its upregulation in the heart after endurance training has not previously been reported.

Previously, exercise training has been shown to improve the oxygen consumption rate of $\mathrm{db} / \mathrm{db}$ cardiomyocytes, but complex specific respiration has not been assessed [28]. Here we show that exercise training specifically improves CII and CIV OXPHOS capacity, but not $\mathrm{Cl}$ OXPHOS capacity. The consequence of a lack of training response in $\mathrm{Cl}$ could be a reduced mitochondrial efficiency. The amount of protons being translocated across the inner mitochondrial membrane is lower with electron input from $\mathrm{Cll}$ than from $\mathrm{Cl}$ [29], which translates to a lower amount of ATP produced per oxygen consumed [30]. Thus, while a potential restoration of maximal $(\mathrm{Cl}+\mathrm{II})$ respiration with exercise training would be beneficial, the mitochondria could be rendered less efficient.

Fragmented mitochondria have been interpreted as a sign of impaired mitochondrial quality control [31], where dysfunctional mitochondrial fragments are not removed by autophagy at a sufficient rate. In heart failure, exercise training ameliorates an impaired autophagic flux and restores mitochondrial size [31]. We noted only a partial recovery in mitochondrial size after exercise training. High glucose levels have been shown to cause mitochondrial fragmentation [32] and reduce mitophagy function [33], suggesting it could drive the changes seen in the $\mathrm{db} / \mathrm{db}$ mice. Serum glucose was still severely elevated in the exercise trained mice which might explain the modest response of mitochondrial morphology to exercise training.

High serum glucose could also drive the reduced $\mathrm{Ca}^{2+}$ response seen in the $\mathrm{db} / \mathrm{db}$ mitochondria. Cardiomyocytes exposed to high glucose show lower MCU protein levels and decreased intramitochondrial $\mathrm{Ca}^{2+}[13]$. Thus, the lack of effect of exercise training on blood glucose could explain why MCU content and mitochondrial $\mathrm{Ca}^{2+}$ response did not significantly improve with exercise training. $\mathrm{Ca}^{2+}$ is released into the cytoplasm during excitationcontraction coupling and instigates contraction, while at the same time entering the mitochondria and activating mitochondrial enzymes [9]. Thus, a reduced $\mathrm{Ca}^{2+}$ response could render the mitochondria less able to properly match energy supply to demand. 
Table 1 Echocardiography

\begin{tabular}{|c|c|c|c|c|c|c|c|c|c|}
\hline \multirow{2}{*}{$\begin{array}{l}\text { Age } \\
\text { Group }\end{array}$} & \multicolumn{3}{|l|}{8 weeks } & \multicolumn{3}{|c|}{16 weeks (after intervention) } & \multicolumn{3}{|c|}{16 weeks (dobutamine) } \\
\hline & Control & T2DM Sed & T2DM Ex & Control & T2DM Sed & T2DM Ex & Control & T2DM Sed & T2DM Ex \\
\hline Heart rate (bpm) Stroke & $416 \pm 25$ & $422 \pm 39$ & $398 \pm 42$ & $448 \pm 32$ & $399 \pm 51^{*}$ & $433 \pm 35$ & $544 \pm 20$ & $499 \pm 41^{*}$ & $509 \pm 22 *$ \\
\hline volume $(\mu \mathrm{l})$ Cardiac & $44.4 \pm 4.7$ & $43.1 \pm 8.4$ & $40.2 \pm 7.6$ & $43.8 \pm 9.8$ & $43.6 \pm 5.2$ & $42.3 \pm 6.2$ & $41.3 \pm 7.1$ & $39.1 \pm 4.6$ & $40.4 \pm 5.3$ \\
\hline $\begin{array}{l}\text { output (ml/min) } \\
\text { Ejection fraction (\%) }\end{array}$ & $\begin{array}{l}18.4 \pm 2.1 \\
64.1 \pm 4.8\end{array}$ & $\begin{array}{l}18.2 \pm 4.2 \\
73.6 \pm 6.8^{*}\end{array}$ & $\begin{array}{l}16.0 \pm 3.5 \\
65.8 \pm 5.1^{\ddagger}\end{array}$ & $\begin{array}{l}19.6 \pm 4.5 \\
69.2 \pm 7.3\end{array}$ & $\begin{array}{l}17.4 \pm 2.8 \\
70.2 \pm 6.3\end{array}$ & $\begin{array}{l}18.4 \pm 3.5 \\
65.5 \pm 4.0\end{array}$ & $\begin{array}{l}22.5 \pm 4.3 \\
84.2 \pm 6.9\end{array}$ & $\begin{array}{l}19.5 \pm 2.7 * \\
81.1 \pm 8.7\end{array}$ & $\begin{array}{l}20.5 \pm 3.0 \\
78.9 \pm 10.3\end{array}$ \\
\hline$E / A$ & $1.5 \pm 0.1$ & $1.4 \pm 0.1$ & $1.5 \pm 0.1$ & $1.5 \pm 0.2$ & $1.7 \pm 0.3$ & $1.6 \pm 0.3$ & $1.5 \pm 0.2$ & $1.6 \pm 0.5$ & $1.5 \pm 0.3$ \\
\hline$E / E^{\prime}$ & $37.9 \pm 9.2$ & $43.9 \pm 7.8$ & $47.9 \pm 12.8$ & $35.5 \pm 8.0$ & $39.9 \pm 7.9$ & $38.1 \pm 16.0$ & $60.0 \pm 20.8$ & $49.9 \pm 13.0$ & $48.3 \pm 15.8$ \\
\hline Mean velocity $(\mathrm{mm} / \mathrm{s})$ & $364 \pm 46$ & $305 \pm 37 *$ & $312 \pm 61$ & $304 \pm 40$ & $341 \pm 97$ & $369 \pm 29$ & $347 \pm 67$ & $334 \pm 82$ & $338 \pm 75$ \\
\hline Peak velocity $(\mathrm{mm} / \mathrm{s})$ & $676 \pm 72$ & $604 \pm 64$ & $615 \pm 118$ & $567 \pm 69$ & $667 \pm 168$ & $700 \pm 47$ & $684 \pm 118$ & $653 \pm 137$ & $669 \pm 125$ \\
\hline
\end{tabular}

${ }^{*} p>0.05$ versus control at the same age, ${ }^{*} p>0.05$ versus T2DM Sed at the same age

The regulation behind the respiratory response to $\mathrm{Ca}^{2+}$ and OXPHOS capacity are likely different. Mitochondria from MCU knockout mice lack responsiveness to $\mathrm{Ca}^{2+}$, yet OXPHOS capacity is the same as in control mice [11]. Therefore, the lack of training-effect on $\mathrm{Cl}$ OXPHOS capacity and the respiratory response to $\mathrm{Ca}^{2+}$ (measured in $\mathrm{Cl}$ ) in the present study are probably not directly linked. Supporting this interpretation, the reduced $\mathrm{Ca}^{2+}$ response is most likely related to the downregulated $\mathrm{MCU}$, which would affect the whole mitochondria. If the lack of exercise training adaptation of $\mathrm{Cl}$ OXPHOS capacity was due to a diminished $\mathrm{Ca}^{2+}$-response, we would probably have seen similar effects in other complexes, not only of $\mathrm{Cl}$. Still, we cannot exclude a common mechanism behind the lack of training response in $\mathrm{Cl}$ to both $\mathrm{Ca}^{2+}$ stimulation and OXPHOS capacity. It is possible that the damage of the ETC complexes, sustained due to T2DM, also left the $\mathrm{db} / \mathrm{db}$ mitochondria less sensitive to calcium. We measured the $\mathrm{Ca}^{2+}$ response during $\mathrm{Cl}$ respiration as other respiratory states (i.e., $\mathrm{Cl}+\mathrm{II}$ ) use oxygen more quickly, making the differentiation between $\mathrm{Ca}^{2+}$ response and the effect of a potential oxygen limitation more difficult.

The modest deteriorations in cardiac function, despite mitochondrial dysfunction, indicate that mitochondrial dysfunction might appear prior to cardiac dysfunction in $\mathrm{db} / \mathrm{db}$ mice. We have previously observed more serious deteriorations in cardiac function at older ages in the $\mathrm{db} / \mathrm{db}$ model [34]. Still, others have found cardiac dysfunction assessed by echocardiography in this animal model before 16 weeks of age [35, 36]. Additionally, the observation that reduced cardiac output in $\mathrm{db} / \mathrm{db}$ only reached significance after dobutamine injection could be connected to the diminished mitochondrial $\mathrm{Ca}^{2+}$ response. The $\mathrm{db} / \mathrm{db}$ mice may have an impaired cardiac response to dobutamine because of the lower MCU content $[11,12]$. The higher EF in the sedentary than exercise trained $\mathrm{db} / \mathrm{db}$ at 8 weeks are likely due to chance since the intervention had not yet begun and the heart rates were similar. All EF values were within normal range and the exact meaning of higher EF in sedentary $\mathrm{db} / \mathrm{db}$ is hard to interpret. One explanation could be that the higher EF could be a sign of compensation due to cardiac remodeling. However, at 16 weeks the difference is smaller and not statistically significant. The EF did not change between the age of 8-16 weeks suggesting that EF is not a sensitive measurement in the window of the intervention.

The upregulation of genes for the Krebs cycle enzymes seems counterintuitive with the reduced mitochondrial function seen in $\mathrm{db} / \mathrm{db}$ mice. In particular for SDH, since $\mathrm{CII}(\mathrm{SDH})$ respiration is reduced in $\mathrm{db} / \mathrm{db}$ hearts. This could indicate that the upregulated gene expression is a compensatory mechanism for reduced mitochondrial function. Also, upregulated expression is not always translated to more protein, and posttranslational modifications could also contribute to decreased function despite increased gene expression.

Reduced cardiac mitochondrial OXPHOS $[8,37]$ and reduced in vivo cardiac function $[22,38]$ has been found in humans as well as in $\mathrm{db} / \mathrm{db}$ mice, indicating that the model is relevant to the heart of humans with T2DM.

\section{Conclusion}

In a mouse model of T2DM, exercise training ameliorates the dysfunction in mitochondrial OXPHOS capacity of complex $\mathrm{II}$ and IV in the heart. Furthermore, the disturbed mitochondrial morphology seen in the $\mathrm{db} / \mathrm{db}$ heart seems to be partially restored with exercise training. The ability of the mitochondria to increase respiration in response to an increased $\mathrm{Ca}^{2+}$ load is impaired in $\mathrm{db} / \mathrm{db}$, but not rescued by exercise training. 
Author Contributions FHB, DC and TOS designed the study, FHB, SS, PC and TOS contributed to data collection. FHB, SS, PC, CM, JS, LHB, MAH, DC, TOS contributed to interpretation of the data and drafting and revising the manuscript.

Funding This work was supported by grants from The Research Council of Norway (FRIPRO Project Number 214458) and (Young Outstanding Investigators Project Number 231764), The Liaison Committee between the Central Norway Regional Health Authority (Project Number 90158300) and UNIKARD (Project Number 217777/H10).

Availability of Data and Material The datasets used and/or analyzed during the current study are available from the corresponding author on reasonable request.

\section{Compliance with Ethical Standards}

Conflict of interest The authors declare that they have no competing interests.

Ethical Approval The study was approved by the Norwegian council for animal research.

\section{References}

1. Collaborators GBDCoD. (2017). Global, regional, and national age-sex specific mortality for 264 causes of death, 1980-2016: A systematic analysis for the Global Burden of Disease Study 2016. Lancet, 390(10100), 1151-1210.

2. Emerging Risk Factors Collaboration. (2010). Diabetes mellitus, fasting blood glucose concentration, and risk of vascular disease: A collaborative meta-analysis of 102 prospective studies. The Lancet, 375(9733), 2215-2222.

3. Haffner, S. M., Lehto, S., Rönnemaa, T., Pyörälä, K., \& Laakso, M. (1998). Mortality from coronary heart disease in subjects with type 2 diabetes and in nondiabetic subjects with and without prior myocardial infarction. New England Journal of Medicine, 339(4), $229-234$.

4. Donahoe, S. M., Stewart, G. C., McCabe, C. H., Mohanavelu, S., Murphy, S. A., Cannon, C. P., et al. (2007). Diabetes and mortality following acute coronary syndromes. JAMA, 298(7), 765-775.

5. Jaffe, A. S., Spadaro, J. J., Schechtman, K., Roberts, R., Geltman, E. M., \& Sobel, B. E. (1984). Increased congestive heart failure after myocardial infarction of modest extent in patients with diabetes mellitus. American Heart Journal, 108(1), 31-37.

6. Belke, D. D., Larsen, T. S., Gibbs, E. M., \& Severson, D. L. (2000). Altered metabolism causes cardiac dysfunction in perfused hearts from diabetic (db/db) mice. American Journal of Physiology Endocrinology and Metabolism, 279(5), E1104-E1113.

7. Dabkowski, E. R., Baseler, W. A., Williamson, C. L., Powell, M., Razunguzwa, T. T., Frisbee, J. C., et al. (2010). Mitochondrial dysfunction in the type 2 diabetic heart is associated with alterations in spatially distinct mitochondrial proteomes. American Journal of PhysiologyHeart and Circulatory Physiology, 299(2), H529-H540.

8. Boudina, S., Sena, S., Theobald, H., Sheng, X., Wright, J. J., Hu, X. X., et al. (2007). Mitochondrial energetics in the heart in obesityrelated diabetes: Direct evidence for increased uncoupled respiration and activation of uncoupling proteins. Diabetes, 56(10), 2457-2466.

9. Glancy, B., Willis, W. T., Chess, D. J., \& Balaban, R. S. (2013).

Effect of calcium on the oxidative phosphorylation cascade in skeletal muscle mitochondria. Biochemistry, 52(16), $2793-2809$.

10. Denton, R. M. (2009). Regulation of mitochondrial dehydrogenases by calcium ions. Biochimica et Biophysica Acta (BBA)Bioenergetics, 1787(11), 1309-1316.

11. Kwong, J. Q., Lu, X., Correll, R. N., Schwanekamp, J. A., Vagnozzi, R. J., Sargent, M. A., et al. (2015). The Mitochondrial calcium uniporter selectively matches metabolic output to acute contractile stress in the heart. Cell Reports, 12(1), 15-22.

12. Rasmussen, T. P., Wu, Y., Joiner, M. L., Koval, O. M., Wilson, N.

R., Luczak, E. D., et al. (2015). Inhibition of MCU forces extramitochondrial adaptations governing physiological and pathological stress responses in heart. Proceedings of the National Academy of Sciences of the United States of America, 112(29), 9129-9134.

13. Diaz-Juarez, J., Suarez, J., Cividini, F., Scott, B. T., Diemer, T., Dai, A., et al. (2016). Expression of the mitochondrial calcium uniporter in cardiac myocytes improves impaired mitochondrial calcium handling and metabolism in simulated hyperglycemia. American Journal of Physiology Cell Physiology, 311(6), C1005-C10c13.

14. Suarez, J., Cividini, F., Scott, B. T., Lehmann, K., Diaz-Juarez, J., Diemer, T., et al. (2018). Restoring mitochondrial calcium uniporter expression in diabetic mouse heart improves mitochondrial calcium handling and cardiac function. Journal of Biological Chemistry, 293(21), 8182-8195.

15. Myers, J., Prakash, M., Froelicher, V., Do, D., Partington, S., \& Atwood, J. E. (2002). Exercise capacity and mortality among men referred for exercise testing. The New England Journal of Medicine, 346(11), 793-801.

16. Wisloff, U., Nilsen, T. I., Droyvold, W. B., Morkved, S., Slordahl, S. A., \& Vatten, L. J. (2006) A single weekly bout of exercise may reduce cardiovascular mortality: how little pain for cardiac gain? 'The HUNT study, Norway'. European Journal of Cardiovascular Prevention and Rehabilitation: Official Journal of the European Society of Cardiology, Working Groups on Epidemiology \& Prevention and Cardiac Rehabilitation and Exercise Physiology, 13(5):798-804.

17. Manson, J. E., Greenland, P., LaCroix, A. Z., Stefanick, M.L., Mouton, C. P., Oberman, A., et al. (2002). Walking compared with vigorous exercise for the prevention of cardiovascular events in women. The New England Journal of Medicine, 347(10), 716-725.

18. Swank, A. M., Horton, J., Fleg, J. L., Fonarow, G. C., Keteyian, S., Goldberg, L., et al. (2012). Modest increase in peak VO 2 is related to 
better clinical outcomes in chronic heart failure patients: Results from heart failure and a controlled trial to investigate outcomes of exercise training. Circulation Heart Failure, 5(5), 579-585.

19. Tjonna, A. E., Lee, S. J., Rognmo, O., Stolen, T. O., Bye, A., Haram, P. M., et al. (2008). Aerobic interval training versus continuous moderate exercise as a treatment for the metabolic syndrome: A pilot study. Circulation, 118(4), 346-354.

20. Wisloff, U., Stoylen, A., Loennechen, J. P., Bruvold, M., Rognmo, O., Haram, P. M., et al. (2007). Superior cardiovascular effect of aerobic interval training versus moderate continuous training in heart failure patients: A randomized study. Circulation, 115(24), 30863094.

21. Hollekim-Strand, S. M., Bjorgaas, M. R., Albrektsen, G., Tjonna, A. E., Wisloff, U., \& Ingul, C. B. (2014). High-intensity interval exercise effectively improves cardiac function in patients with type 2 diabetes mellitus and diastolic dysfunction: A randomized controlled trial. Journal of the American College of Cardiology, 64(16), 1758-1760.

22. Stølen, T. O., Høydal, M. A., Kemi, O. J., Catalucci, D., Ceci, M., Aasum, E., et al. (2009). Interval training normalizes cardiomyocyte function, diastolic $\mathrm{Ca}^{2+}$ control, and SR Ca ${ }^{2+}$ release Synchronicity in a Mouse Model of Diabetic cardiomyopathy. Circulation Research, 105(6), 527-536.

23. Shao, C. H., Wehrens, X. H., Wyatt, T. A., Parbhu, S., Rozanski, G. J., Patel, K. P., et al. (2009). Exercise training during diabetes attenuates cardiac ryanodine receptor dysregulation. Journal of Applied Physiology, 106(4), 1280-1292.

24. Wang, H., Bei, Y., Lu, Y., Sun, W., Liu, Q., Wang, Y., et al. (2015). Exercise prevents cardiac injury and improves mitochondrial biogenesis in advanced diabetic cardiomyopathy with PGC-1alpha and Akt activation. Cellular Physiology and Biochemistry, 35(6), 21592168.

25. Coleman, D. L., \& Hummel, K. P. (1967). Studies with the mutation, diabetes, in the mouse. Diabetologia, 3(2), $238-248$.

26. Kemi, O. J., Loennechen, J. P., Wisløff, U., \& Ellingsen, $\varnothing$ (2002). Intensity-controlled treadmill running in mice: Cardiac and skeletal muscle hypertrophy. Journal of Applied Physiology,

93(4), 1301-1309.

27. Ruas, J. L., White, J. P., Rao, R. R., Kleiner, S., Brannan, K. T., Harrison, B. C., et al. (2012). A PGC-1alpha isoform induced by resistance training regulates skeletal muscle hypertrophy. Cell, 151(6), 1319-1331.

28. Veeranki, S., Givvimani, S., Kundu, S., Metreveli, N., Pushpakumar, S., \& Tyagi, S. C. (2016). Moderate intensity exercise prevents diabetic cardiomyopathy associated contractile dysfunction through restoration of mitochondrial function and connexin 43 levels in $\mathrm{db} / \mathrm{db}$ mice. Journal of Molecular and Cellular Cardiology, 92, 163-173.

29. Hinkle, P. C., Kumar, M. A., Resetar, A., \& Harris, D. L. (1991).

Mechanistic stoichiometry of mitochondrial oxidative phosphorylation. Biochemistry, 30(14), 3576-3582.

30. Brand, M. D., Harper, M. E., \& Taylor, H. C. (1993). Control of the effective P/O ratio of oxidative phosphorylation in liver mitochondria and hepatocytes. Biochemical Journal, 291(Pt 3),

739-748.

31. Campos, J. C., Queliconi, B. B., Bozi, L. H. M., Bechara, L. R. G., Dourado, P. M. M., Andres, A. M., et al. (2017) Exercise reestablishes autophagic flux and mitochondrial quality control in heart failure. Autophagy. https://doi.org/10.1080/15548627.2017.1325062.

32. Yu, T., Robotham, J. L., \& Yoon, Y. (2006). Increased production of reactive oxygen species in hyperglycemic conditions requires dynamic change of mitochondrial morphology. Proceedings of the National Academy of Sciences of the United States of America, 103(8), 2653-2658.

33. Devi, T. S., Somayajulu, M., Kowluru, R. A., \& Singh, L. P. (2017). TXNIP regulates mitophagy in retinal Muller cells under high-glucose conditions: Implications for diabetic retinopathy. Cell Death \& Disease, 8(5), e2777.

34. Stolen, T. O., Hoydal, M. A., Kemi, O. J., Catalucci, D., Ceci, M., Aasum, E., et al. (2009). Interval training normalizes cardiomyocyte function, diastolic $\mathrm{Ca}^{2+}$ control, and SR $\mathrm{Ca}^{2+}$ release synchronicity in a mouse model of diabetic cardiomyopathy. Circulation Research, 105(6), 527-536.

35. Semeniuk, L. M., Kryski, A. J., \& Severson, D. L. (2002).

Echocardiographic assessment of cardiac function in diabetic $\mathrm{db} / \mathrm{db}$ and transgenic $\mathrm{db} / \mathrm{db}-\mathrm{hGLUT4}$ mice. American Journal of Physiology Heart and Circulatory Physiology, 283(3), H976-H982.

36. Venardos, K., De Jong, K. A., Elkamie, M., Connor, T., \& McGee, S. L. (2015). The PKD inhibitor CID755673 enhances cardiac function in diabetic db/db mice. PLOS ONE, 10(3), e0120934.

37. Anderson, E. J., Kypson, A. P., Rodriguez, E., Anderson, C. A., Lehr, E. J., \& Neufer, P. D. (2009). Substrate-specific derangements in mitochondrial metabolism and redox balance in atrium of type 2 diabetic human heart. Journal of the American College of Cardiology, 54(20), 1891-1898.

38. Palmieri, V., Bella, J. N., Arnett, D. K., Liu, J. E., Oberman, A., Schuck, M. Y., et al. (2001). Effect of type 2 diabetes mellitus on left ventricular geometry and systolic function in hypertensive subjects: Hypertension Genetic Epidemiology Network (Hyper-GEN) study. Circulation, 103(1), 102-107.

Publisher's Note Springer Nature remains neutral with regard to jurisdictional claims in published maps and institutional affiliations.

\section{Affiliations}

Fredrik H. Bækkerud ${ }^{1}$. Simona Salerno ${ }^{1} \cdot$ Paola Ceriotti $^{2,3} \cdot$ Cecilie Morland $^{4} \cdot$ Jon Storm-Mathisen ${ }^{5}$. Linda H. Bergersen $^{6,7} \cdot$ Morten A. Høydal ${ }^{8,9} \cdot$ Daniele Catalucci ${ }^{2,3} \cdot{\text { Tomas O. St } \varnothing l e n^{1,8,9}}^{2}$

Simona Salerno simona.salerno@ntnu.no

Paola Ceriotti paoletta.ceriotti@gmail.com

Cecilie Morland cecilie.morland@farmasi.uio.no 
Jon Storm-Mathisen jon.storm-mathisen@medisin.uio.no

Linda H. Bergersen I.h.bergersen@odont.uio.no

Morten A. Høydal morten.hoydal@ntnu.no

Daniele Catalucci daniele.catalucci@cnr.it

Tomas O. Stølen tomas.stolen@ntnu.no

1 Department of Circulation and Medical Imaging, Faculty of Medicine and Health Science, K.G. Jebsen Centre of Exercise in Medicine, Norwegian University of Science and Technology, Postboks 8905, 7491 Trondheim, Norway

2 Institute of Genetics and Biomedical Research, Milan Unit, National Research Council, 20138 Milan, Italy

3 Humanitas Clinical and Research Center, Rozzano, 20089 Milan, Italy

4 Department of Pharmaceutical Biosciences, School of Pharmacy, University of Oslo, Oslo, Norway

5 Division of Anatomy, Department of Molecular Medicine, Institute of Basic Medical Sciences, CMBN/SERTA Healthy Brain Ageing Centre, University of Oslo, Oslo, Norway

6 Department of Oral Biology, University of Oslo, Oslo, Norway

7 Department of Neuroscience and Pharmacology, Center for Healthy Aging, University of Copenhagen, Copenhagen, Denmark

8 Group of Molecular and Cellular Cardiology, Department of Circulation and Medical Imaging, Faculty of Medicine and Health Science, Norwegian University of Science and Technology, Trondheim, Norway

9 St. Olavs University Hospital, Trondheim, Norway 